\title{
The Development of the Ideas and Perspectives of the Joined Wing Aircraft
}

\author{
Semenov Vladimir ${ }^{1,2}$, Phone Myint Tun ${ }^{1}$, Voityshen Vladimir ${ }^{1,2}$ and Hardeep Zinta ${ }^{1}$ \\ ${ }^{1}$ Moscow Institute of Physics and Technology, Department of Aeromechanics and Flight Engineering, \\ 140180, Gagarina Street, 16, Zhukovsky, Moscow Region, Russia \\ ${ }^{2}$ Central Aerohydrodynamic Institute (TsAGI). 140180, Zhukovsky Street, 1, Zhukovsky, Moscow Region, Russia
}

\begin{abstract}
The history of the development constructive aircraft scheme with a joined wing, the main sphere areas of their use and advantages in characteristics. Prospects for the application of a joined-wing scheme in adaptive constructions. The development of actuators based on shape memory alloys.
\end{abstract}

\section{Introduction}

In 1910 years, the designer L. Bleriot used the scheme of an aircraft with a joined wing for his aircraft Bleriot III (Fig.1).

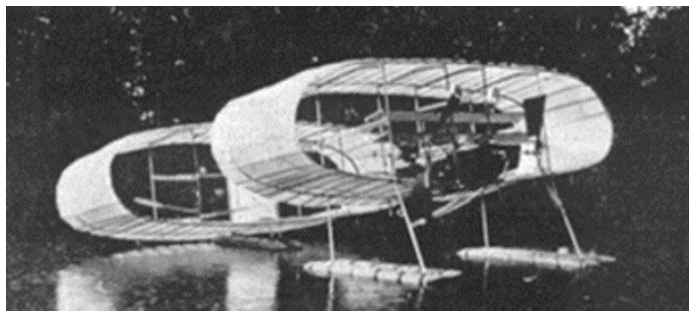

Figure 1. Bleriot III, 1906

In the 1920 years received priority development biplanes. In the 1930 years, for speed aircraft the main place in aviation took monoplane aircraft scheme.

The biplane and the joined wing, which are sometimes mistakenly identified, are fundamentally different schemes. So, in biplane big building height constructions created on account of numerous racks and bracelets, working as rod elements, and in a joined scheme of the wings the elements work with the transfer of moment forces, and this creates favorable prerequisites for upgrade the characteristics aircraft, in the first queue, in the field of strength and weight reduction of the wing design.

In 1968 years, J. Wolkovitch wrote fundamental article [1], which compared aircraft with a monoplane and joined wing. This article substantiated the potential advantages of an aircraft with a joined wing (Fig.2) in many disciplines, including aerodynamics, flight control, strength and rigidity, specific weight of the structure.

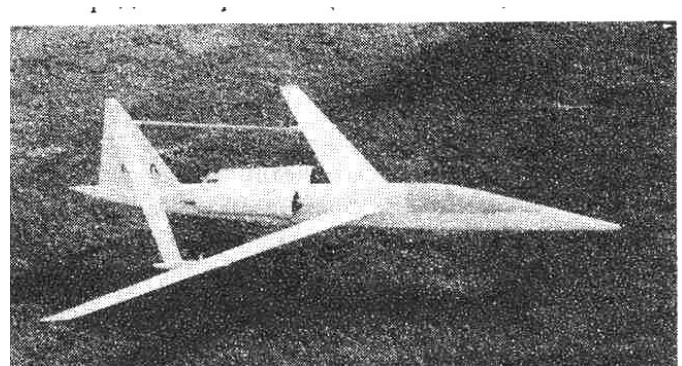

Figure 2. Model by J. Wolkovitch. US. 1985

It was shown that in the transition from a monoplane to a joined wing design, in conditions of conservation the equivalent aerodynamic characteristics, weight of the power part wing design can be reduced by $20 \div 30 \%$, maximum deflections of the wing reduced by $2 \div 3$ times.

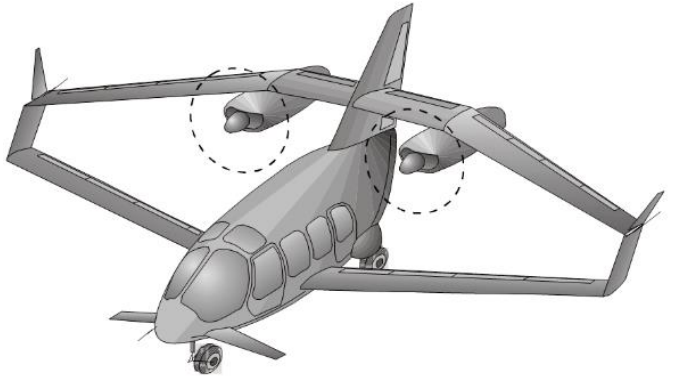

Figure 3. Aircraft "Stalker-232”. 1996

Research, conducted in the 1990 years on the design aircraft "Stalker-232" (A.P. Rudometkin, Kramatorsk, Ukraine; V.N. Semenov, Yu.S. Mikhailov, TsAGI, Russia) confirmed also the possibility direct control of the lifting and lateral force aircraft by combination of flap deflection on the elements of the wing system, having a significant transverse V-imagery (Fig.3). 
In articles [2,3] received and recommended relationships of geometry parameters joined wing, ensuring reduction its weight on condition ensuring strength, and also compared the aerodynamic properties. The next fundamental research was completed in Russia 2001 years in research work (scientific research work), performed at the Moscow Aviation Institute (MAI) together with other enterprises [4]. There were compared two aircraft concepts: with a classic monoplane wing (Fig.4), and with a joined wing (Fig.5).

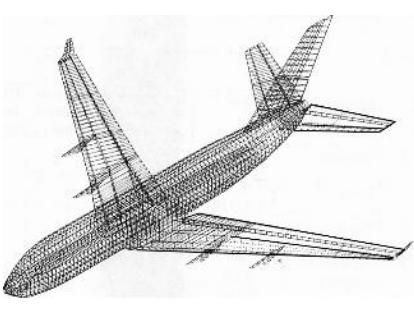

Figure 4. Monoplan;

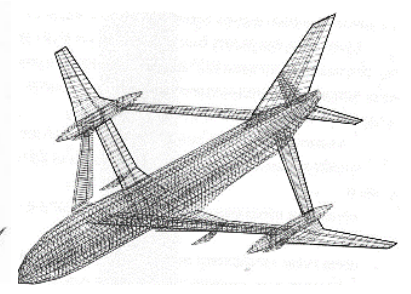

Figure 5. Joined wing by V.V. Malchevsky
The last two schemes, despite significant technical advantages before the first two, have problems with leaving the plane passengers in emergencies, and require a principle different design concept and long-term flight practice for development of new technical solutions, and also place increased demands on the runways.

Table 1. Comparative characteristic of schemes of aircrafts designed for the same requirement:

$$
\text { Npas }=600, L=13000 \mathrm{~km}, M=0,85
$$

\begin{tabular}{|l|c|c|}
\hline $\begin{array}{r}\text { Scheme } \\
\text { Characteristics }\end{array}$ & \multirow{2}{*}{$\begin{array}{c}\text { Monoplan } \\
\text { (fig. 4) }\end{array}$} & $\begin{array}{c}\text { Joined wing } \\
\text { (fig. 5) }\end{array}$ \\
\hline Lift-to-drag ratio & \multirow{2}{*}{$\begin{array}{c}\text { Initial } \\
\text { version for }\end{array}$} & $-4,0 \%$ \\
\cline { 1 - 1 } \cline { 1 - 1 } Weight of empty & \begin{tabular}{c} 
comparison \\
\cline { 1 - 1 } Fuel mass
\end{tabular} & $-16,5$ \\
\cline { 1 - 1 } Take-off mass & $(100 \%)$ & $-10,5$ \\
\hline
\end{tabular}

The world's Aircraft building companies continue research and the design of an aircraft in a joined-wing scheme $[5,6]$. Despite the advantages of a joined wing, their level is until insufficient for achievement large-scale economic advantages, such a level, so that air carriers began to order such planes, and accordingly, were made investments in the restructuring of factories and their equipment. Require additional arguments, demonstrating significant advantages on special requirements for the project.

\section{Theoretical basis of weight gain in the joined wing design}

In the figure 6 show that in a joined wing created by external forces bending moments $M$ in each section are balance by the sum of the moments, acting in the upper $\left(M_{1}\right)$ and the lower $\left(M_{2}\right)$ wings, and moments, determined by the compression forces of the upper wing $N_{l}$, and stretch of the lower wing $N_{2}$ taking into account the shoulders of their applications $\left[h_{l}\right.$ and $\left.h_{2}\right]$.

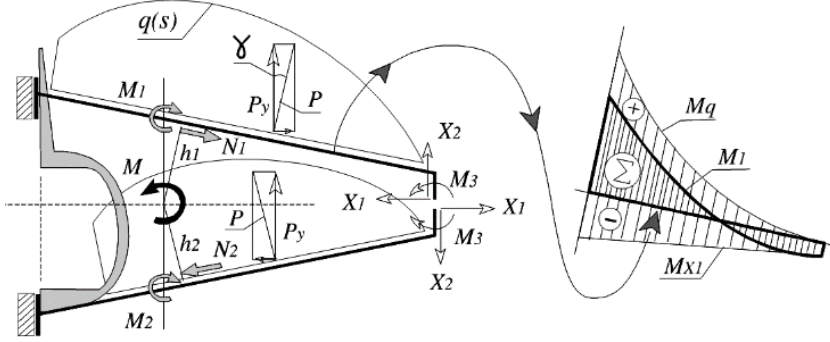

Figure 6. Superposition of bending moments

This equation can be written in the form of an equation of equilibrium:

$$
-M=M_{1}+M_{2}+N_{2} h_{1}+N_{2} h_{2}
$$

In the figure 6 it is also shown (on the right) that in the element of a statically indeterminate frame due to the superposition of bending moments from external load $M_{q}$ and the internal force of interaction of wing elements on the end washer $M_{x l}$, their total moment $M_{l}$ is significantly reduced.

Specific material costs, going on the formation of elements, working on bending, as a rule, much higher, then for elements, which reaction structure are represented by a pair of forces. In real constructions are present both specified forms perception of bend. In view of this, if by constructive transformations or changing the spatial shape of constructions manage increase the fraction of moments, perceived in the form of effort tension-compression, and respectively, reduce moments in elements, working on bending, possible to achieve a reduction mass in constructions and reduce the level of deformation. That is, at unchanged left side of equation (1) there is possible to change ratio of the first and the second pair of terms in right-hand side, and then higher the proportion of the second pair, more rational constructions. In the joined wings system, consisting from rectilinear sections, manage to $45 \div 55 \%$ bending moment from an external load to equilibrate efforts tensile-compression in the wings, spaced on high altitude, and thereby reduce the weight of the corresponding force elements.

Weight of the materials construction, going perception $M_{b e n d}$, can be estimated as an integral from the area of the corresponding diagram, taken module:

$$
G=\frac{k \gamma}{[\sigma]} \int_{L} \frac{M_{x}(z) d z}{h_{z}}
$$

Where $[\sigma]-$ level permissible stresses,

$\gamma$ - Specific weight of the structural material,

$h_{z}$ - averaged caisson height in section $z$,

$L$ - half-size of the wing line,

$k$ - Statistical coefficient, accounting mass non structure components of the wing. Approximately $k=2$. 
This follows, what is the changing shape of the elastic axis of the joined wing, we change the value of the total moment in its section. Cross-section, in which acts the longitudinal force $N$, offset by the value $\Delta y$ from the original rectilinear elastic axis, causes an additional compensation moment $N^{*} \Delta y$, which promotes to reduce the weight of the wing. Finally, belt of spars are in different conditions of superposition load, and redistribution of power material between them gives another direction of optimization.

\subsection{Constructions with a curved elastic axis wing}

In the publication $[5,6]$ it was proposed to give the elastic axis of the joined wing wave shape, minimize the required structural weight of the wing. Methods and program are developed, finding the optimal deviation of the elastic axis of the wing from its original linear shape.

In figure 7 presents shape guise of the aircraft with optimized by criteria Strength and weight of the shape of the elastic axis of the joined wing.

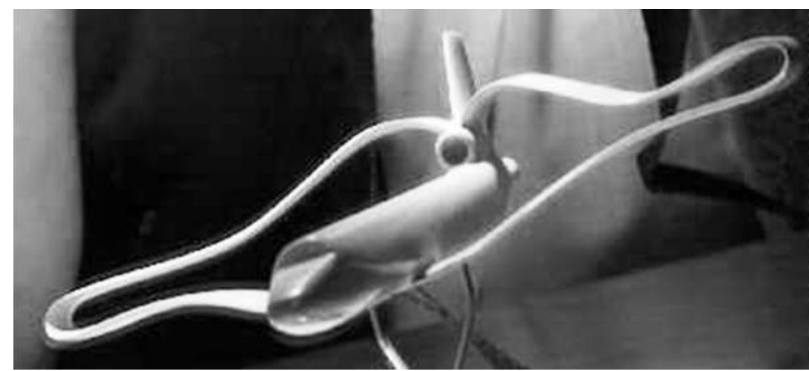

Figure 7. Aircraft by patent Ru N 2067948.

V.N. Semenov, V.V. Saurin. TsAGI, 1996

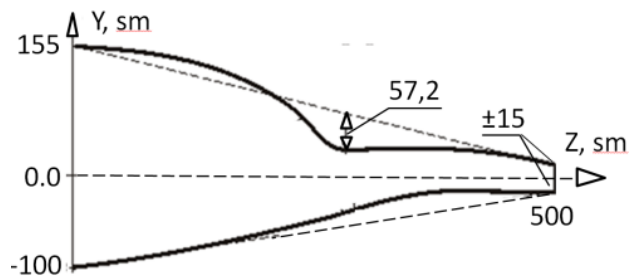

Figure 8. Optimal form of the elastic axes of a joined wing

In figure 8 presented rational curvilinear form of the elastic axes of a joined wing. It is not symmetrical relative to the horizontal plane in connected with geometric constraints, superimposed on the lower wing. Wing half-size $500 \mathrm{~cm}$, height in the plane of symmetry $255 \mathrm{~cm}$, the maximum deviation of the axis from the original position is equal to $-57,2 \mathrm{~cm}$.

Table 2. The vertical deviation of the optimal elastic axes from the original linear shape.

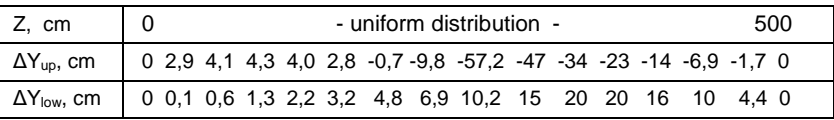

\subsection{Structure influence shape of the axis joined wing aircraft on its weight}

Mass of a classic monoplane wing approximately consider as consisting their two parts. Origins optimized force mass (weight) is taken as $100 \%$. During the course of the synthesis shape of the elastic axis due to the joined contour managed to reduce wing weight by $25 \%$ and another $15 \%$ for calculate and finding the optimal curvilinear axis. This reducing weight is calculated by changing in the internal load structure.

At the same time in weight gain increases by changing the geometry of the wing. $4 \%$ adds an increase in the "linear" length of the wing axis due to the growth of transverse V-shape, and 8\% - due to its curvilinear.

From statistical data it is known, that in the wing design there is present also a mass, on which optimization has no effect, and this fragment of mass is practically equal to the original wing effort. Therefore, in the total balance of the wing mass, the gain is approximately $14 \%$.

\subsection{Search for the optimal shape of the joined wing axis on a flat model}

In the figure 9 shown 3D-model of the project of a convertiplane with a joined wing in flight. Turn engine is carried out around axes - actuators, manufactured from shape memory alloy, which are continuous twisted element. Show the scale is the attachment height of the root section of the rear wing and the point for variation of the height of the elastic axis, position of which are parametrically change vertically to obtain a piecewise linear solution.

Investigation search optimal shape of the elastic wing axis, carried out on a flat model aircraft in the form of a flight. At this accounted for only the main factor load, influencing on weight $-M_{\text {bend, }}$ and also, that the stiffness of the wing box in the plane bending for two order less, than in the direction of flight.

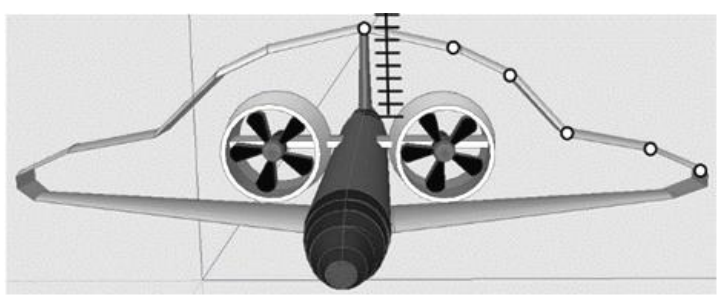

Figure 9. The 3D-model of convertiplane

At figure 10 compare the variants of linear and piecewise linear elastic wing axes, allow to reduce weight of a power part of a design of a wing on 23 and $39 \%$ respectively. When more complete accounting all force factors and cases of load, these profits are usually reduced. 


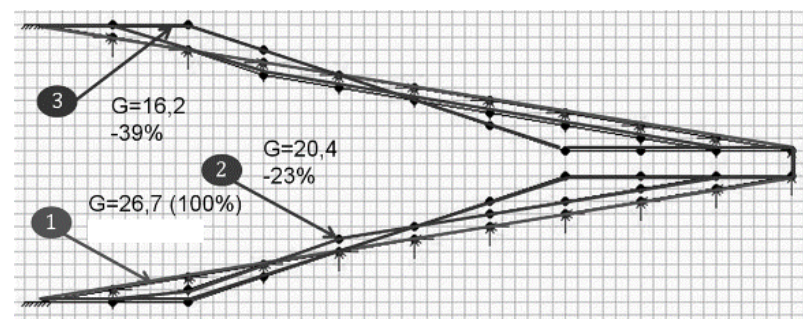

Figure 10. Variants of the forms of the elastic axes of a joined wing

At figure 11 comparison of the upper wing bending moments $M_{\text {bend }}$ for variants 1 and 3 for the joined wing of the model (Fig.9). On the lower wing, not shown parts of beams, diagrams $M_{\text {bend }}$ skew-symmetric the upper wing.

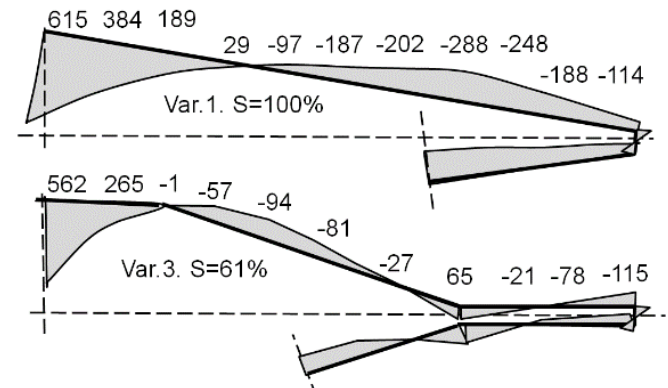

Figure 11. Comparison of the upper wing bending moments

\subsection{Eigen forms and Eigen frequencies of an aircraft with a joined wing}

There was performed modal analysis of a prospective aircraft with takeoff weight 45 tons and span of the joined wing - $28 \mathrm{~m}$. The elastic axis of the joined wing consisted of straight sections. For analysis was created a FEM model of aircraft which was consist of the beam elements. This model had the stiffness and masses distributions along the wing and fuselage, which were obtained on the basis of airworthiness for Static Strength.
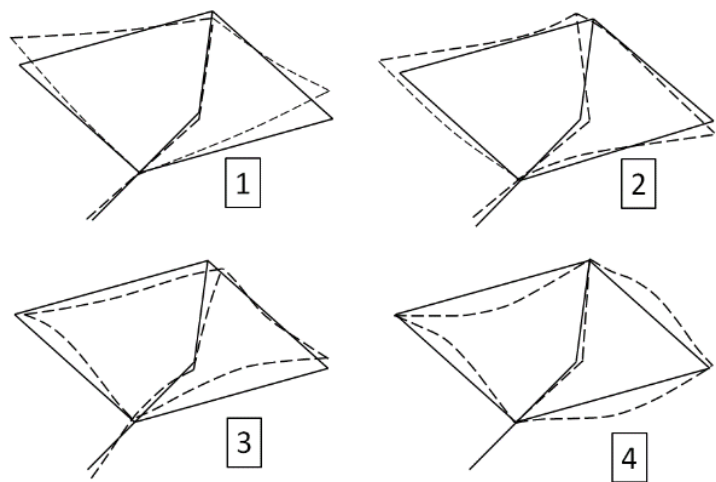

Figure 12. The first four Eigen forms of aircraft

At figure 12, are shown the first four Eigen forms of aircraft in order of increasing Eigen frequencies: 2.14, $3.32,4.92,6.85 \mathrm{~Hz}$ respectively. Of these, two forms are symmetrical (first and third) and two - anti symmetrical (second and fourth). The solid line represents the elastic axes of the undeformed aircraft.
Study has shown, that with increasing rigidity of the joined wing the Eigen frequency of the aircraft are increased, which positively affects the reserves at the critical speed of the flutter of the aircraft.

Changing the Eigen frequencies after optimization of the design by the criteria of static strength and the minimum weight has a different orientation, and can as create problems, so and help in solving problems of dynamic strength.

\section{Shape memory alloys}

The aircraft have many flight regimes and accordingly to calculated loading cases, each of has its own rational configuration. The transformation of the aircraft structure using mechanical drives makes the fragments of aircraft split and adds weight to it. Ideal option is, when the design itself change its configuration and perform selfadjustment, remaining in the state of a single continuous body. Shape memory alloy effect can achieve this possibility [7-9]. The shpe memory alloy consists of two or more phases (austenitic, martensitic, rhombohedral, and others) with different mechanical properties. Unlike conventional composites, shape memory alloys components can transform into each other with varying temperature and (or) stresses (thermoelastic martensitic phase transformations) or change the structure of the crystal lattices of some of the components under the action of stresses (structural transition). As a result, the shape memory alloys can accumulate or return deformations of large magnitude, that's why, it can be controlled to change its shape. Thanks to the phase and structural transitions occurring in the shape memory alloys, these materials have unique thermomechanical properties. They are characterized by such phenomena as accumulation of strains of direct transformation, monotonous and reversible shape memory, martensitic inelasticity and superelasticity, oriented transformation. The variety of unique properties of the shape memory alloys predetermines the broad prospects of their use for the construction of unique design with adaptive, intelligent and other new properties.

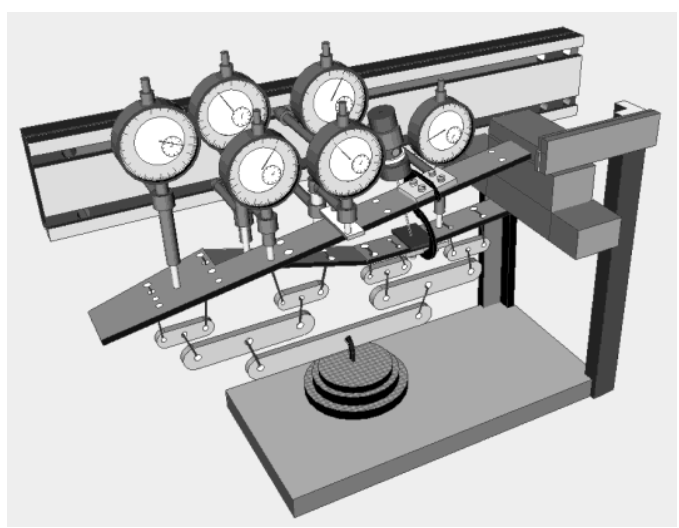

Figure 13. The 3D model of the stand for study of the joined wing

At the Faculty of Aeromechanics and Flight Engineering, MIPT, work is under way to demonstrate the capabilities of the actuators made from shape memory 
alloys for the transformation and control of structural deformations. The possibility of changing the angle of attack of a joined wing using an actuator is being studied. At the stand shown in figure 13, the model of the joined wing of the aircraft is affected by an $S$-shaped element made of a NiTi wire of $3 \mathrm{~mm}$. This element is installed at the half-span of the joined-wing element system, and simulates a washer-actuator. Heating the puck leads to its extension, with the appearance of large forces deforming the joined wing. The local stress-strain state is investigated by a SPECKLE-holographic installation.

As a result of the action of the actuator, the angle of attack of the wing system increases by several degrees, which depends on the span from the conditions of closing the root sections of the wing, and the proportion of the cantilever part. Thus, it is possible to change the integral angle of attack of the aircraft wing relative to the axis of its fuselage. When the heater is removed from the actuator, the stiffness of the swirling joined-wing system serves as a return spring and brings it to its original position. A wire made of $T N-1$ alloy (titanium nickelide) was used. The temperatures of the process of the martensitic transitions of the alloy are taken as follows: $M n=57^{\circ} \mathrm{C}, M k=17^{\circ} \mathrm{C}, A n=67^{\circ} \mathrm{C}, A k=107^{\circ} \mathrm{C}$.

Shape memory alloys properties allow creating devices that realize complex kinematics of deformation displacement of structural elements with high weight return of devices, their constructive simplicity and location in the minimum volume.

The problem in the technical implementation of massive devices from shape memory alloys is the difficulty in ensuring their rapid and even uniform heating (cooling), since the non-simultaneous start of the martensitic transition of the crystal structure in different layers of the device leads to the occurrence of large internal stresses, and this can lead to the appearance of cracks.

\subsection{Design ratios for the NiTi (nickel - titanium shape memory alloys ) tubular actuator}

To solve the problems of adaptation of aircraft to the flight regime, tubular torsion actuators are considered to be the most acceptable, and at the same time they act as load-bearing elements of the structure, for example, sections of the tubular wing spar, or the axis of the whole-feather tail [6-9].

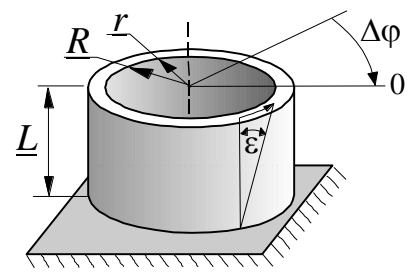

Figure 14.The cross-section parameters of the active element

The radius of the cross-section of the active element $R$ (Fig. 14) is selected from the condition for providing the required operating torque $\mathrm{M}$ of the drive [7]:

$$
R \geq \sqrt[3]{\frac{2 \sqrt{3} M}{\pi \sigma\left(1-\frac{r^{4}}{R^{4}}\right)}}
$$

Where $L$ - is the length of the working cylinder,

$D$ - is the diameter,

$\varepsilon-$ is the relative pseudo plastic deformation.

For the NiTi alloy, $\varepsilon<5 \div 10 \%$

$\varphi$ - torsion angle $360^{\circ}$ rotation of reversible torsional strain is achieved with a $L / D$ ratio of about 10 .

(for $90^{\circ} L=2.5 \mathrm{D}$ )

The following relations and restrictions are also used:

$$
\begin{aligned}
& \varepsilon=D \varphi / 4 L \\
& L / R \geq \varphi / \sqrt{ }(3 \varepsilon) \\
& \varepsilon^{*}<(4 \div 8) \%, \\
& \sigma^{*}<(400 \div 800) \mathrm{MPa} .
\end{aligned}
$$

The problem in the technical implementation of massive devices from shape memory alloys is the difficulty in ensuring their rapid cooling.

\subsection{To the synthesis of biologically similar structures}

Direct axes and regularity of structural elements underlie many aircraft projects. Often one can see an analogy in the decisions that nature and science have found. One of the promising areas of the development of aircraft designs is related to the science of Bionics, which studies the possibility of using the principles of organization, properties, functions and structures of living nature in technical devices and systems. When using bionic solutions, it is first important to identify the principal possibility of implementing a particular idea, and then, on the basis of certified methods, to prove its rationality. The monoplane trapezoidal wing shown in figure 15 is calculated and the weight of its structure is taken as 1 .

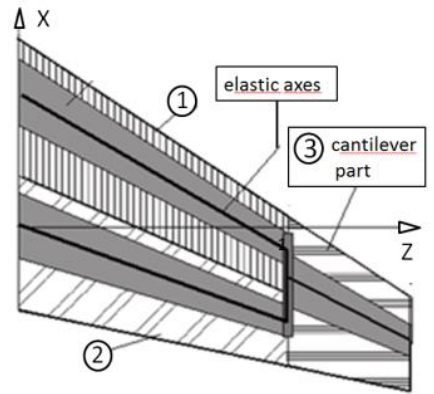

Figure 15. Equivalence of the areas of the reference monoplane wing and joined wing

Further, the monoplane wing is transformed into a joined one, consisting of a cantilever tip part and two planes, the root parts of which are spaced in height in the projection in the direction of flight, as shown in figure 16. 


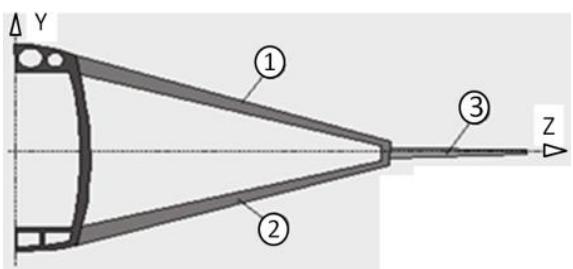

Figure 16. The monoplane wing is transformed into a joined wing, consisting of a two planes root parts 1,2 , and cantilever tip part 3

The figure 17 shows how the weight of the wing depends on the share of its cantilever part.

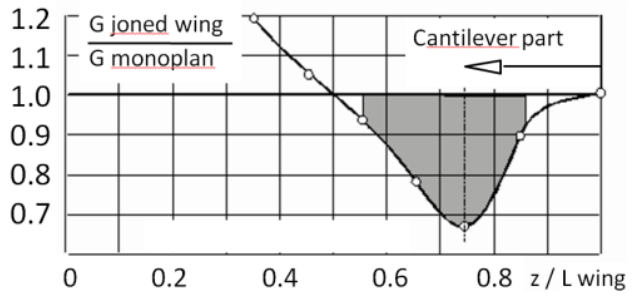

Figure 17. Dependence of wing weight from its cantilever portion size

Obtained during the synthesis of the structure by the criterion of its minimum weight, the shape of the aircraft with a joined system of wings with a cantilever part was naturally similar to the element of the spine of the Katran (the Black Sea shark) shown in figure 18.

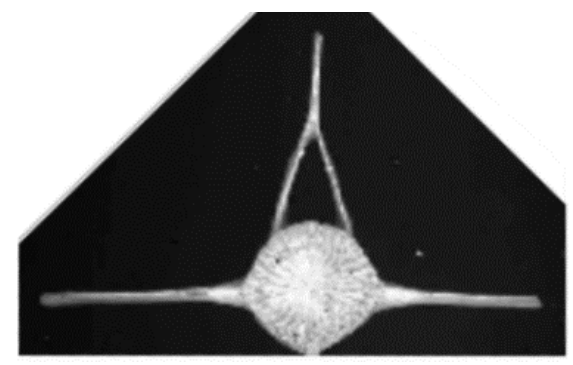

Figure 18. Shark's vertebra - a biological analogue of a joined wing with a cantilever part

\section{Adaptive layout aircrafts}

Adaptive designs that adjust their configuration to flight mode and aircraft loading have great prospects for improving technical and economic characteristics. In the past decades, the main progress was achieved through multi-criteria optimization of structures made from materials with permanent properties. As our knowledge and technological capabilities expand, the concept of design includes an ever wider range of tasks to be solved.

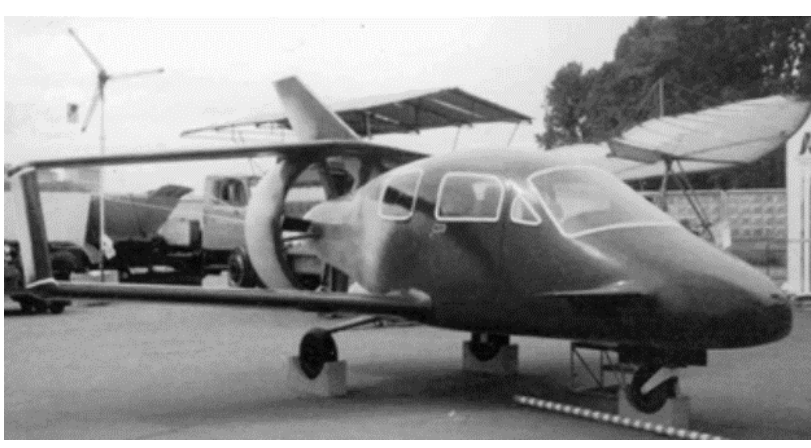

Figure 19. Airplane by V.S. Jaeger "E-1". MAKS- 2000

A whole-composite aircraft was constructed (Fig. 19) for which the technology of continuous winding of highstrength filaments in a joined contour of wings was developed.

The project is considered, in which the wing of the convertiplane is twisted in the takeoff and landing modes like the wing of a bird (Fig. 20, 21). The main problem is to find an economical way to convert the geometry of the wing. One of the solutions is the use of shape memory alloys compartments integrated monolithically in a continuous cylindrical spar. To solve the problems of adaptation of the aircraft to the flight mode, tubular torsion-active actuators are considered to be the most acceptable, and at the same time they serve as loadbearing elements of the structure, for example, sections of the tubular wing spar, or the axis of the whole-feather tail.

Conversion of the aircraft to a new optimal working configuration, in the transition from takeoff and landing regimes to flight configurations, can be achieved by turning the propellers relative to the fuselage of aircraft, or by changing the mutual arrangement of aggregates, until they are completely joined, changing the sweep angles and twisting the wing, changing the length and the twist of the propeller blades and other transformations [69].

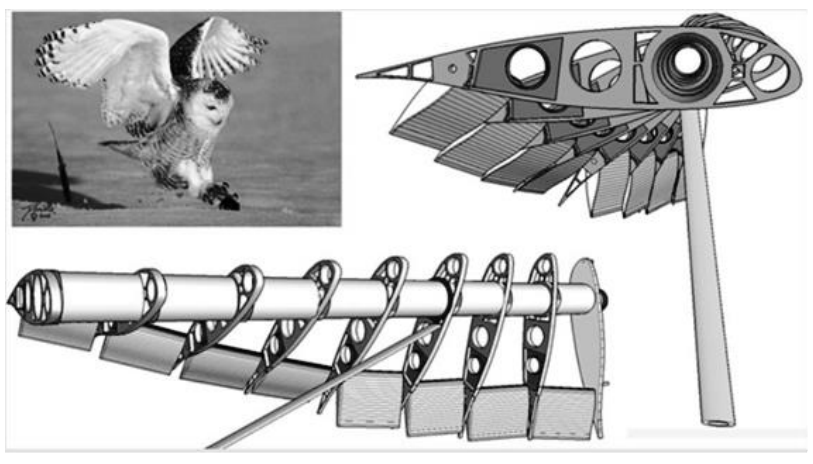

Figure 20. Deformation mechanism, making movements similar to the wing of a bird

Figure 20 shows the structure of the adaptive wing using the tubular compartments of the spar from shape memory alloys. The spar member between adjacent ribs has a section of shape memory alloys capable of making an autonomous rotation at a predetermined angle and a passive region of traditional metal. 
Heating the corresponding rings from the shape memory alloys makes it possible to form any form of twist of the compartments, as shown in figure 20,21. It is important to show specialists in aerodynamics and flight control of aircrafts on the promising realizability of such transformations, which can initiate their search for the rational use of adaptation of the shape of the structure.

The mechanical and functional properties of the shape memory alloys make it possible to form on their basis intelligent devices that react by their behavior to changes in the external environment.

The insertion of elements from the shape memory alloys into joined strong structure makes it possible to redistribute the shear flows into the structure, to act on deformations, to create, when necessary, large internal forces, and also to be an energy accumulator.

The use of shape memory alloys makes possible to construct adaptive constructions with gapless kinematics which is actual for a convertiplanes. The combination of these methods allows you to make advancement in the idea of creating aircraft with solar traction, and drones with a record low relative weight of the structure.

In accordance with the equation of the existence of an aircraft, the gain in the specific weight of the structure makes it possible to use this gain in any other components of the total weight of the aircraft: improving aerodynamics by increasing the wing extension, payload weight and taking additional fuel.

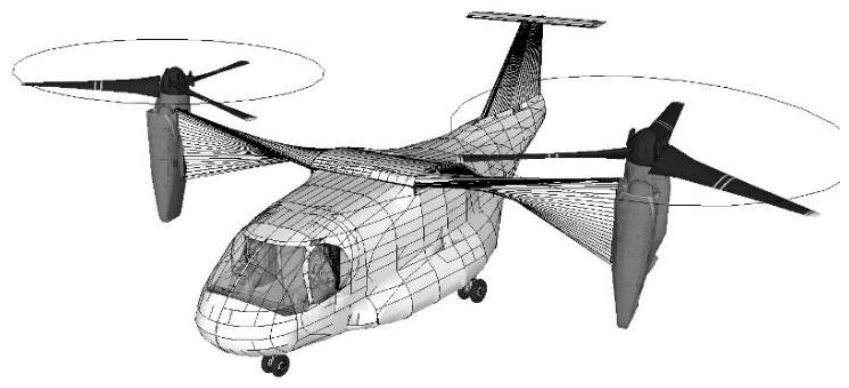

Figure 21. The convertiplane with sectional folded spars

One of the promising methods for creating complex structures with internal cavities is 3D printing. However, at the present time, the required dimensions of the products have not yet been achieved, and the continuity of the material being stacked and its required characteristics are not always ensured.

Aircraft with joined wings can be especially effective when used as high-altitude, working on accumulated solar energy. Especially large lengthening of the wing leads to their great flexibility and deformations. In this case, a joined system of aircraft substructures with a large building height is the most important factor preventing the destruction of the structure.

For an aircraft with a joined wing, rational proportions of the design parameters have already been formed. Thus, the transverse $\mathrm{V}$ of the wing is optimal at \pm $15 \div 17^{\circ}$, and the share of the console of the joined wing system, if present, is about $25 \%$ of the wing's half-span.

The idea of using a joined wing in aircraft designs from the field of science has already passed to the stage of design and practical use. The deterrent is still the high intensity of science projects and the lack of proven prototypes and well-proven technical solutions.

\section{References}

1 J. Wolkovitch. The Joined Wing: An overview.// Journal of Aircraft, v. 23, No. 3, 1986. P. 161-178.

2 V.N. Semenov. A comparison of the weight ration of the airframe designs of aircraft with a cantilever wing and with a joined wing system. // NASA TM77842. Apr.1985.

3 S.V. Novoseltsev, V.N. Semenov. Investigation of the aerodynamic and strength characteristics of an aircraft with a joined biplane system of wings // M., TVF, No. 7, 1984.

4 V.V. Malchevsky. The design of very large commercial transport aircraft with nontraditional configuration, ISTS 501-98. Moscow 2001.

5 V.V. Saurin, V.N. Semenov. Optimization of the shape of a joined beam structure under a distributed load. TsAGI, No. 3, 1992. P. 85-93.

6 V.N. Semenov. Constructions of airplanes of joined and variable circuits. TsAGI, 2006. 228 p.

7 A.A. Movchan, Co Nyunt, V.N. Semenov Designing a torque exciter for an alloy with shape memory. // Proceedings of TsAGI. 2004. Issue. 2664. P. 220-230.

8 I.E. Vyakhi, V.N. Semenov. The use of alloys with shape memory as drives and active elements of adaptive designs of aircraft. // Proceedings of TsAGI. 2005. Issues. 1996. P. 208-214.

9 V.S. Voityshen, M.Yu. Kollerov, V.N. Semenov, V.N. Shcherbakov, Phone Myint Tun. Tendencies of using alloys with shape memory in engineering and medicine. // Mechanics of composite materials and structures. Moscow: IPRIM RAS. T.23. No. 2. 2017. P. 157-167. 